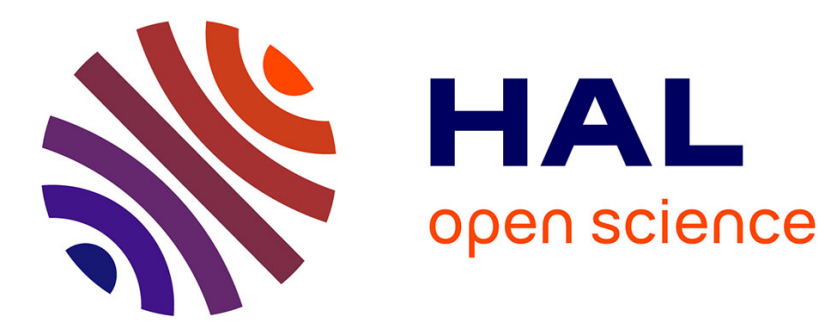

\title{
Test of the formal basis of Arrhenius law with heat capacities
}

\author{
Denis Michel
}

\section{To cite this version:}

Denis Michel. Test of the formal basis of Arrhenius law with heat capacities. 2018. hal-01786914

\section{HAL Id: hal-01786914 \\ https://hal.science/hal-01786914}

Preprint submitted on 7 May 2018

HAL is a multi-disciplinary open access archive for the deposit and dissemination of scientific research documents, whether they are published or not. The documents may come from teaching and research institutions in France or abroad, or from public or private research centers.
L'archive ouverte pluridisciplinaire HAL, est destinée au dépôt et à la diffusion de documents scientifiques de niveau recherche, publiés ou non, émanant des établissements d'enseignement et de recherche français ou étrangers, des laboratoires publics ou privés. 


\title{
Test of the formal basis of Arrhenius law with heat capacities
}

\author{
Denis Michel ${ }^{\ddagger}$
}

$\ddagger$ Universite de Rennes1-IRSET. Campus de Villejean. 35000 Rennes France. Email: denis.michel@live.fr.

\begin{abstract}
The exponential factor of Arrhenius satisfactorily quantifies the energetic restriction of chemical reactions but is still awaiting a rigorous basis. By assuming that the Arrhenius equation should be grounded on statistical mechanics and is probabilistic in nature, two structures are compared for this equation, depending on whether reactant energies are envisioned as the mean values of specific energy distributions, or as particular levels in a global energy distribution. In the first version, the Arrhenius exponential factor would be a probability depending once on temperature while in the second one it is a ratio of probabilities, depending twice on temperature. These concurrent equations are tested using experimental data for the isomerization of 2-butene. This comparison reveals the fundamental structure of the Arrhenius law in isothermal systems and overlooked properties resulting from the introduction of reactant energies in the equation.
\end{abstract}

Keywords: Kinetics; equilibrium; heat capacity; enthalpy.

The celebrated laws of Arrhenius and van't Hoff are the basements of teaching and research in physical chemistry. Various versions of the energetic restriction of reactions have been proposed before the currently used Arrhenius equation, which works pretty well but remains empirical [1]. A mathematical approach will be used here to ground these equations on rational bases. The probabilistic tool dedicated for this purpose is likely to be the geometric or exponential distribution, which proved sufficient to recover Boltzmann statistics [2, 3].

\section{Exponential distribution of en- ergy}

Contrary to the historical equations of Arrhenius and van't Hoff, the new relations described here between energy, kinetic and equilibrium constants will be based on Boltzmann's laws of energy distribution. More precisely, the exponential distribution underlies both Boltzmann theory and the thermochemical constants. Indeed, to define the distribution of maximal randomness in mechanical systems, mathematics provides an unambiguous shortcut bypassing Hamiltonians and Lagrangians: this is necessarily the "memoryless" exponential distribution [2]. A probabilistic distribution relies upon a unique function called probability density function (PDF). That of the exponential law is simply

$$
f(\mathcal{E})=\frac{1}{\langle\mathcal{E}\rangle} \mathrm{e}^{-\frac{\mathcal{E}}{\langle\mathcal{E}\rangle}}
$$

where $\langle\mathcal{E}\rangle$ is the mean value of the distribution. We can apply it to energy distribution by supposing that $\mathcal{E}$ is the number of energy units in a particle. This function monotonously decreasing towards high energies is illustrated in Fig.1, which represents the densities of particles containing different amounts of energy. The low energy particles are the most numerous and give the dark lower levels, whereas the particles are less and less numerous when going up in the energy levels. Probabilities are obtained by integration of the PDF. The probability that a randomly picked particle has an energy equal to or higher than a certain threshold $\mathcal{E}^{\ddagger}$ is

$$
P\left(\mathcal{E} \geqslant \mathcal{E}^{\ddagger}\right)=\int_{\mathcal{E}=\mathcal{E}^{\ddagger}}^{\infty} f(\mathcal{E}) d \mathcal{E}=\mathrm{e}^{-\frac{\mathcal{E}^{\ddagger}}{\langle\mathcal{E}\rangle}}
$$

Note that the double dagger $(\ddagger)$, taken again here, is historical in the field of rate theories to symbolize the energy threshold. To be validated by statistical mechanics, this approach should also allow recovering Boltzmann's distribution. This requirement is fully satisfied since the probability that a particle has an energy level precisely of $\mathcal{E}^{\ddagger}$, can indeed be obtained by integration of the same 
PDF, but between $\mathcal{E}^{\ddagger}$ and $\mathcal{E}^{\ddagger}+1$.

$$
\begin{aligned}
P\left(\mathcal{E}=\mathcal{E}^{\ddagger}\right) & =\int_{\mathcal{E}=\mathcal{E}^{\ddagger}}^{\mathcal{E}^{\ddagger}+1} f(\mathcal{E}) d \mathcal{E} \\
& =\left(1-\mathrm{e}^{-\frac{1}{\langle\mathcal{E}\rangle}}\right) \mathrm{e}^{-\frac{\mathcal{E}^{\ddagger}}{\langle\mathcal{E}\rangle}} \\
& =\frac{\mathrm{e}^{-\frac{\mathcal{E}^{\ddagger}}{\langle\mathcal{E}\rangle}}}{\sum_{j=0}^{\infty} \mathrm{e}^{-\frac{j}{\langle\mathcal{E}\rangle}}}
\end{aligned}
$$

The latter form of Eq.(3) is known as the partition function of Boltzmann, recovered here without need for the usual introductory treatments of statistical mechanics. Eq.(2), that is less familiar in thermochemistry, quantifies the probability for a particle of exceeding a threshold energy. It could evoke the formula of Arrhenius

$$
k=A \mathrm{e}^{-\frac{E_{a}}{k_{B} T}}
$$

but this is only apparent because as it is structured, the Arrhenius equation cannot be a probability 3. To use Eq.(2) for constructing the energy restriction formula of rate constants, we must first agree on the nature of the average energy $\langle\mathcal{E}\rangle$. Identifying $\langle\mathcal{E}\rangle$ as $k_{B} T$ allows to use the exponential law described above to recover in a simple manner different extensions of statistical mechanics, including energy densities and Maxwell velocity densities 3]; but for an application to chemistry where the different molecules have different energies, the nature of $\langle\mathcal{E}\rangle$ should be precised. Two formulas are possible depending on whether the interconvertible species belong to the same distribution or to two different distributions 3 .

\subsection{Hypothesis of molecule type-specific energy distributions}

Each type of molecule, with a specific name and defined by chemists on the basis of its covalent architecture, has its own specific energy which could be envisioned as its average energy. Within a homogeneous population of such a type of molecule, the energy of each individual molecule could take different values because of thermal fluctuations. For example, a double bond $\mathrm{C}=\mathrm{C}$ could be more or less rotated or stretched. This view is illustrated in Fig.1A, where $E_{i}$ and $E_{j}$ are mean energies corresponding to different values of $\langle\mathcal{E}\rangle$ in Eq.(2), apart of which individual molecules can take different energy levels. The probability that a given molecule has an energy higher than a threshold $\mathcal{E}^{\ddagger}$ is exactly described by
Eq.(2). As the two interconvertible species have their own average energy, this hypothesis will be called Model II. The extensions of this model have been thoroughly described in [3].

\subsection{Hypothesis of the general mean en- ergy}

In this second option illustrated in Fig.1B, all molecules, regardless of their chemical classification, participate to a global energy distribution, characterized by a single average energy value. In this unique continuum of chemical energies, the covalent architecture used for the classification of molecules, has no more importance than secondary phenomena like bond twisting and stretching. As it is based on a unique energy distribution, this hypothesis will be called Model I.

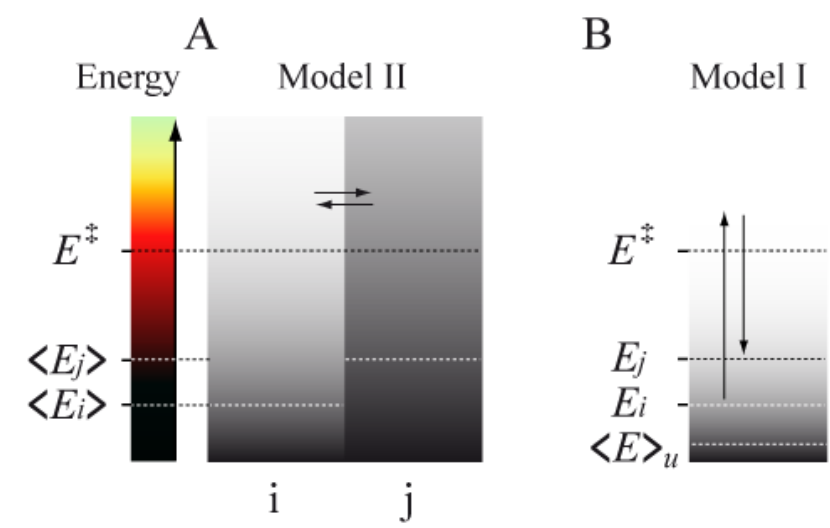

Figure 1. Competing views of the energy restriction of rate constants. The quantities written in brackets are average values of exponential distributions, whereas the quantities written without brackets are particular energy levels belonging to these distributions. (A) A mean energy value is assigned to each type of molecule $i$ or $j$. (B) The energies of the different types of molecules are not mean values but fixed values belonging to a common energy distribution with a general mean value $\langle E\rangle_{u}$.

\section{Application of these assump- tions to the Arrhenius formula}

The two views of mean energies described above will be applied to the following generic form of rate constant

$$
k=A_{(T)} \mathrm{e}^{B_{(T)}}
$$

which is, like the Arrhenius equation, made of a preexponential and an exponential term, both depending on temperature. The preexponential factor $A$ does not contribute to the reactional energy of the molecule but corresponds to a configurational recurrence frequency, that 
is to say the frequency at which reappears one of the $\varpi$ molecular configurations favorable to the reaction, out of a total number $\Omega$ of possible configurations.

$$
A_{(T)}=\Phi T
$$

with $\Phi=\varpi k_{B} /(\Omega h)$, where $k_{B}$ and $h$ are the Boltzmann and Planck constants respectively. This preexponential factor will no longer be studied here because the different theories more or less explicitly agree on its nature, including the activated complex theory [3]. By contrast, the meaning of the exponent of Arrhenius depends on the theories.

\section{$2.1 \quad$ Model II}

If the different types of reactants are assumed to have specific mean energies, the exponential term of Arrhenius would be the probability that a molecule of type $j$ of mean energy $\left\langle E_{j}\right\rangle$, reaches a threshold $E^{\ddagger}$. This probability is predicted by the exponential distribution.

$$
k_{j i} / A_{j i}=P\left(E \geqslant E^{\ddagger}\right)=\mathrm{e}^{-\frac{E^{\ddagger}}{\left\langle E_{j}\right\rangle}}(T)
$$

The dependence on temperature of this exponent is mediated by the mean energy $\left\langle E_{j}\right\rangle_{(T)}$ only, because the threshold $E^{\ddagger}$ is fixed and temperature-independent. When temperature increases, $\left\langle E_{j}\right\rangle_{(T)}$ increases while $E^{\ddagger}$ is unchanged, so that the reaction rate expectedly increases. In this respect, this theory is in perfect agreement with the traditional interpretation of the stimulatory role of temperature on reaction rates, through a shift of the whole Maxwell-Boltzmann distribution towards higher energies. As a consequence of this shift, the fractional particle population whose energy exceeds a certain threshold, increases. When defining the equilibrium constant as a ratio of such rate constants, the van't Hoff equation reads

$$
K_{j i}=\frac{A_{j}}{A_{i}} \mathrm{e}^{E_{i j}^{\ddagger}\left(\frac{1}{\left\langle E_{i}\right\rangle_{(T)}}-\frac{1}{\left\langle E_{j}\right\rangle_{(T)}}\right)}
$$

which also depends on temperature through the mean energies.

\section{$2.2 \quad$ Model I}

In the unique chemical energy continuum of overall mean value $\langle E\rangle_{u(T)}$ illustrated in Fig.1B, the reactional energies $E_{i}(T)$ and $E_{j}(T)$, as well as the threshold energy $E^{\ddagger}$, are just particular energy levels. According to Eq.(3),

$$
\begin{gathered}
P\left(E=E_{i}\right)=\mathrm{e}^{-\frac{E_{i}}{\langle E\rangle_{u}}}\left(1-\mathrm{e}^{-\frac{1}{\langle E\rangle_{u}}}\right) \\
P\left(E=E_{j}\right)=\mathrm{e}^{-\frac{E_{j}}{\langle E\rangle_{u}}}\left(1-\mathrm{e}^{-\frac{1}{\langle E\rangle_{u}}}\right)
\end{gathered}
$$

and

$$
P\left(E=E^{\ddagger}\right)=\mathrm{e}^{-\frac{E^{\ddagger}}{\langle E\rangle_{u}}}\left(1-\mathrm{e}^{-\frac{1}{\langle E\rangle_{u}}}\right)
$$

In the usual conditions of chemistry, the concentration ratios of molecules with different energies are simply given by their relative probabilities [3, so that the Arrhenius equation can now be defined as

$$
k_{j i} / A_{j i}=\frac{P\left(E=E^{\ddagger}\right)}{P\left(E=E_{i}\right)}=\mathrm{e}^{-\frac{E^{\ddagger}-E_{i}}{\langle E\rangle_{u}}}
$$

A ratio of probabilities, contrary to a probability, can range from 0 to infinity, like the Arrhenius equation. As the same holds for the reciprocal reaction, in equilibrium the concentration ratio between the interconvertible reactants, or equilibrium constant, gets rid of the energy threshold.

$$
\begin{aligned}
K_{j i} & =\frac{A_{j}}{A_{i}} \frac{P\left(E=E^{\ddagger}\right)}{P\left(E=E_{i}\right)} \frac{P\left(E=E_{j}\right)}{P\left(E=E^{\ddagger}\right)} \\
& =\frac{A_{j}}{A_{i}} \frac{P\left(E=E_{j}\right)}{P\left(E=E_{i}\right)} \\
& =\frac{A_{j}}{A_{i}} \mathrm{e}^{\frac{E_{j}-E_{i}}{\langle E\rangle_{u}}}
\end{aligned}
$$

In both hypotheses, the equilibrium distribution of the interconvertible molecules depends primarily on their energy difference, but under the hypothesis that the different molecules have all their own mean energy, this difference is further accentuated by the height of the energy barrier. The model of the generalized chemical continuum conforms to the principle of thermochemical equilibrium in that the energy threshold does not intervene in equilibrium. For this reason it appears simpler, but 
conversely its temperature dependence is more complex and this complexity is overlooked. Indeed, the exponent of rate constants depends twice on the temperature, contrary to what is suggested in the Arrhenius equation expressed as a straight line.

$$
B_{i(T)}=-\frac{E^{\ddagger}-E_{i(T)}}{\langle E\rangle_{u(T)}}
$$

and that of the equilibrium constants depends three times on the temperature

$$
B_{i(T)}-B_{j(T)}=\frac{E_{j(T)}-E_{i(T)}}{\langle E\rangle_{u(T)}}
$$

In this model, the access of a molecule to the energy threshold $E^{\ddagger}$ is favored by an increase in temperature in two ways: (i) by increasing the general average populational energy, which increases the denominator; and (ii) by increasing the specific energy of the considered reactant molecule, which decreases the numerator. The first mechanism is general and applies to all molecules in the isothermal medium, whereas the second mechanism depends on each type of molecule.

\subsection{Theoretical shapes of the Arrhenius plots in the two models}

The energy of reactants, called enthalpy in thermochemistry, is rooted in statistical mechanics, but it results from the combination of many interfering mechanisms particularly complex at low temperature. For simplicity, let us imagine a theoretical reactant consisting of a single resonator of frequency $\nu$ (i.e. absorbing a single wavelength, contrary to the more advanced treatment of Debye), like an Einstein solid. In that respect, it may not be a coincidence if in his original article on heat capacities, Einstein took as a model the diamond 4, which contains only one type of chemical bond (C-C). The discrete approach to thermal mean energy of Planck can be rapidly recovered starting from the number of ways to distribute $E$ energy quanta in $N$ particles

$$
\Omega=\frac{(N+E-1) !}{(N-1) ! E !}
$$

giving a single particle mean entropy of

$$
\mathcal{S}=\frac{1}{N} \ln \Omega=\left(1+\frac{E}{N}\right) \ln \left(1+\frac{E}{N}\right)-\frac{E}{N} \ln \left(\frac{E}{N}\right)
$$

The ratio $E / N$ is equivalent to the mean number of energy quanta per particle $U / h \nu$ [5].

$$
\mathcal{S}=\frac{S}{k_{B}}=\left(1+\frac{U}{h \nu}\right) \ln \left(1+\frac{U}{h \nu}\right)-\frac{U}{h \nu} \ln \left(\frac{U}{h \nu}\right)
$$

Introducing temperature through the fundamental entropy equation

$$
\frac{d S}{d U}=\frac{1}{T}
$$

yields

$$
\frac{k_{B}}{h \nu} \ln \left(1+\frac{h \nu}{U}\right)=\frac{1}{T}
$$

and

$$
U=\frac{h \nu}{\mathrm{e}^{h \nu / k_{B} T}-1}
$$

where $h \nu$ can also be defined as $h \nu=h c / \lambda$, where $c$ is light velocity and $\lambda$ is the spectroscopic wavelength of absorption. Hence, the number of energy quanta used in $\operatorname{Eqs}(1-3)$, is

$$
\langle\mathcal{E}\rangle=\frac{U}{h \nu}=\frac{1}{\mathrm{e}^{h \nu / k_{B} T}-1}
$$

As the heat capacity of a substance is its ability to increase its energy with temperature, it can be derived from Eq.(12b) as did 4,

$$
C=\frac{d U}{d T}=\frac{(h \nu)^{2}}{k_{B} T^{2}} \frac{\mathrm{e}^{h \nu / k_{B} T}}{\left(\mathrm{e}^{h \nu / k_{B} T}-1\right)^{2}}
$$

The shape of this function is represented in Fig.2A, which shows that increasing either the temperature or the wavelength, symmetrically increases heat capacity. The two models described above predict that the Arrhenius equation is not a straight line.

\subsubsection{Model I}

In the theory of the general mean energy, even without introducing the complexity of real heat capacities, the theoretical Arrhenius plot drawn for a single frequency is not linear. Taking $k_{B} T$ as the mean energy, Eq.(8a) becomes

$$
\ln (k / A)=-\frac{E^{\ddagger}}{k_{B} T}+\frac{\frac{h \nu}{k_{B} T}}{\mathrm{e}^{\frac{h \nu}{k_{B} T}}-1}
$$




\subsubsection{Model II}

If neglecting the logarithmic temperature dependence of the preexponential factor, replacing the energy in Eq.(5) by that of Eq.(12b) gives,

$$
\ln k / A=-\frac{E^{\ddagger}}{h \nu}\left(\mathrm{e}^{\frac{h \nu}{k_{B} T}}-1\right)
$$

whose Taylor series is

$$
\ln k / A=\frac{E^{\ddagger}}{h \nu}\left[1-\sum_{j=0}^{\infty}\left(\frac{h \nu}{k_{B} T}\right)^{j} / j !\right]
$$

which can be limited to its first two terms when $1 / T$ and $\nu$ are small enough to render the higher order terms negligible

$$
\ln k / A=-\frac{E^{\ddagger}}{k_{B} T}-\frac{E^{\ddagger} h \nu}{2\left(k_{B} T\right)^{2}}
$$

This second term can generate convex Arrhenius plots for high frequency reactants (Fig.2C). Note that interestingly, the first term of Eq.(16b) is close to the traditional slope when $H$ is negligible compared to $E^{\ddagger}$. In the traditional interpretation, the Arrhenius slope is considered constant, which misleadingly suggests that the Arrhenius plot is a straight line. We see that both theories predict that Arrhenius plots are globally curved. In addition, these curvatures can be further complicated by irregularities of heat capacities, because these simplified plots hold for single resonators, whereas real molecules contain numerous juxtaposed resonators, absorbing at different wavelengths and participating in the global energy of the molecule in a cooperative manner. Hence, molecular energies cannot be determined in a bottom-up approach starting from known wavelengths, but they can be deduced by a reverse strategy from the measured heat capacities.
A

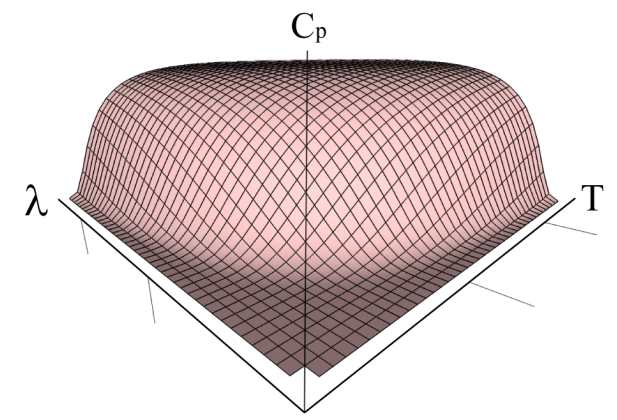

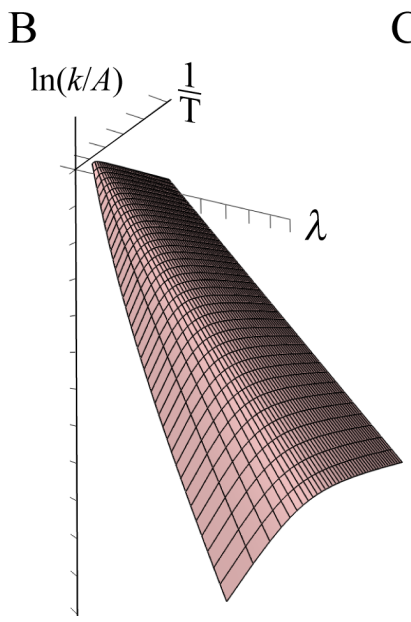

Model I
$\mathrm{C}$

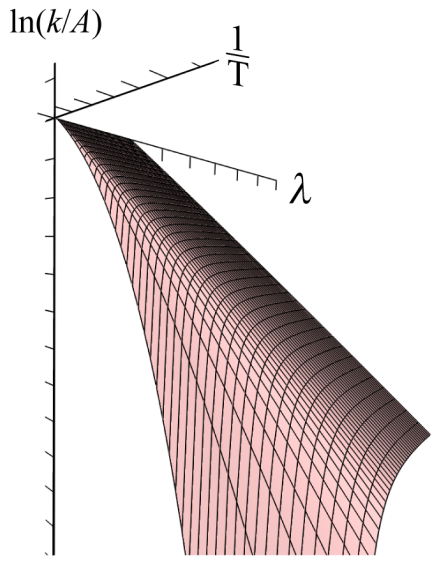

Model II
Figure 2. Minimalist modeling of heat capacity and Arrhenius plots for a theoretical reactant made of a single resonator. (A) Heat capacity symmetrically increases when increasing either temperature or $\lambda$. (B) Shape of Arrhenius plot obtained from the energy continuum hypothesis (Eq.(14)), concave at high reactant frequency. (C) Shape of Arrhenius plot with the hypothesis of a mean reactant energy (Eq.(15)), convex at high reactant frequency. The energy thresholds are arbitrary and the temperature-dependence of the preexponential factor is not taken into account in these representations.

\section{Experimental comparison of the approaches}

In physics, theories are not chosen for their elegance but for their capacity to describe experimental observations. The chemical reaction selected for this test is the isomerization between cis-2-butene $(Z)$ and trans-2-butene $(E)$, because it has a series of advantages: it involves reciprocal reactions unimolecular in both directions. Very precise data are available in kinetics [6] and in equilibrium 7]. Moreover, the thermal isomerization of 2-butene is restricted by a quite high energy barrier (corresponding essentially to the energy needed to break the $\pi$ bond), which should make it possible to clearly distinguish the two models described above, since the barrier plays a role 
in equilibrium in the former but not in the latter. Because of this barrier, the thermal conversion between the two isomers is not measurable at temperatures lower than $700 \mathrm{~K}$. As a consequence, equilibrium data were obtained in presence of a catalyst [7. Finally, the temperaturedependent heat capacities of these two isomers are also available, which will allow to approximate their energies.

\section{Determination of absolute en- ergies}

The two forms of the exponential factor compared here require knowledge of the absolute energies of reactants. The probabilistic form based on reactant-specific average energies needs this value for the denominator of the exponent (Eq.(5)) while the form based on the energy continuum model needs it at the exponent numerator (Eq.(8a)). But absolute energies and enthalpies are notoriously difficult to determine.

\subsection{What form of energy to use}

The energy of the single resonator reactant of frequency $\nu$ used previously is theoretical and not available for determining the molecular energy of Eq.(12b), but the principle of this type of energy will however be retained here, that is to say an energy equal to zero at $T=0 \mathrm{~K}$, increasing with temperature and calculable as the integral of heat capacity, as theorized by Einstein [4] (Eq.(13)). This point is essential since absolute energies are unknown while heat capacities are often known, so we have first to explicit their temperature-dependence in the form of an integrable function. In the energy continuum model similar to the current treatment of thermochemistry, absolute enthalpies $(H)$ will be used, but enthalpies can take negative values, which would lead to a division by zero at a certain temperature in $\mathrm{Eq}(8 \mathrm{a})$, so only positive energies (written $E$ ) will be used for the Model II. Absolute enthalpies can in principle be obtained from heat capacities using the Kirchhoff approach

$$
H(T)=H\left(T_{0}\right)+\int_{T_{0}}^{T} C_{p}(T) d T
$$

where $T_{0}$ is temperature of reference chosen for convenience. The first step is to determine a function satisfactorily adjusted to the heat capacities measured by the Thermodynamics Research Center [8] and available in the NIST Chemistry WebBook. The 3 parameter exponential association

$$
C_{p} \approx \alpha\left(\beta-\mathrm{e}^{-\gamma T}\right)
$$

is chosen here as a fitting function because it is easily integrable and valid for a wide temperature range over $300 \mathrm{~K}$. The fitting parameters are, for the cis isomer $\alpha=264.49, \beta=1$ and $\gamma=13.35 \times 10^{-4}$ (curve shown in Fig.3A), and for the trans isomer $\alpha=266.07, \beta=0.9968$ and $\gamma=12.97 \times 10^{-4}$.
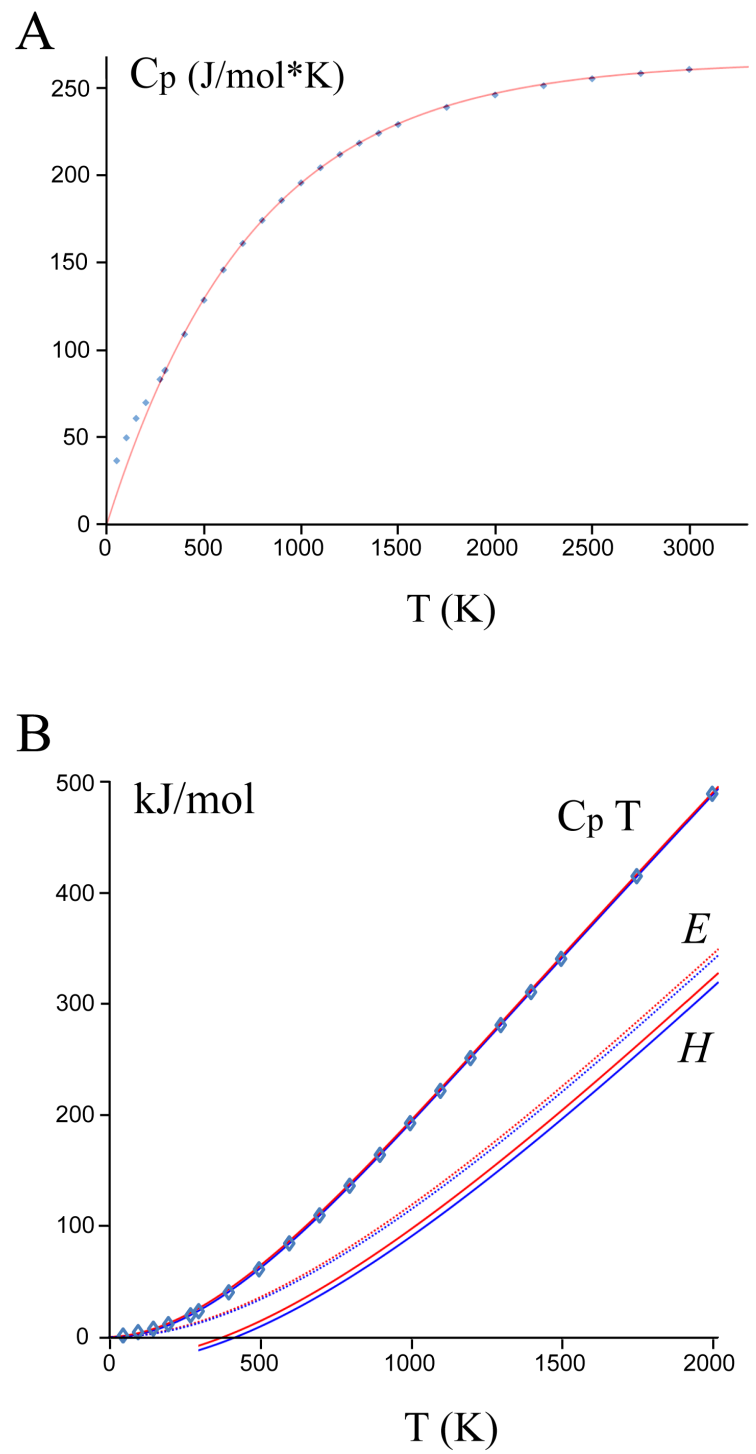

Figure 3. (A) Measured heat capacities and fitting function valid for high temperatures. (B) Absolute enthalpies $(H$, plain lines) and positive energies $(E$, dotted lines) of the 2-butene isomers, red for the cis isomer and blue for the trans isomer. For comparison, energies calculated as direct products $T C_{p}(T)$ are shown (diamonds corresponding to trans-2-butene).

The indefinite integrals $F(T)$ of these functions can then be used to calculate absolute enthalpies provided the enthalpy of formation is known at a given tempera- 
ture $T_{0}$.

$$
H(T)=F(T)-F\left(T_{0}\right)+H_{f}\left(T_{0}\right)
$$

If the starting elements are taken as $0 \mathrm{~J} / \mathrm{mol}$, the values given in the NIST webBook are, for the cis isomer $H_{f Z}(298)=-7.7 \mathrm{~kJ} / \mathrm{mol}$ and for the trans isomer is $H_{f E}(298)=-10.8 \mathrm{~kJ} / \mathrm{mol}$. 9]. To link these values to the range of validity of the exponential fitting curve, the intermediate range is covered by a polynomial approximation (Appendix). The enthalpies found in this way are presented in Fig.3A. For comparison, the values $T C_{p}(T)$ are drawn in the same graph (Fig.3B) to show that this direct approach overestimates the energies.

Based on the view of molecular energy as a positive number of energy quanta, like in Eq.(12b), and to avoid division by zero in the probabilistic approach, internal energies will be simply defined as

$$
E(T)=F(T)-F(0)
$$

The models can now be compared numerically with the kinetic results.

\section{Determination of energy threshold from the Arrhenius slope at $T=800 \mathrm{~K}$}

The rate equations of cis trans isomerization of 2-butene, determined experimentally [6] are

$$
k_{Z \rightarrow E}=9 \times 10^{13} \times \operatorname{Exp}\left(-\frac{31845}{T}\right) s^{-1}
$$

and

$$
k_{Z \rightarrow E}=21 \times 10^{13} \times \operatorname{Exp}\left(-\frac{32725}{T}\right) s^{-1}
$$

The slopes are expressed here in Kelvin units to eliminate a presupposed model. Written in this form, these equations are purely experimental and modelindependent, so they can be used as templates to calculate the parameters of the model-derived equations. Giving to the central experimental temperature $800 \mathrm{~K}$ the best degree of confidence, the parameters are adjusted such that at this temperature, the slope $d(\ln k) / d(1 / T)$ of the theoretical equations gives the experimental slope. The superposition of the resulting curves to the Arrhenius plots of 6], confirms the concavity of Model I (Fig.4A) and convexity of Model II (Fig.4B), both predicted theoretically (Fig.2). Introducing energies in the Arrhenius equation should yield the same value for $E^{\ddagger}$ by two different calculations starting from the two reciprocal reactions.

\section{$5.1 \quad$ Model I}

The two types of energies will be applied to this model.

\subsubsection{Using absolute enthalpies}

We find, for the cis isomer,

- $A_{Z}=2.82 \times 10^{4} s^{-1}$

- $E^{\ddagger}=185.85 \mathrm{~kJ} / \mathrm{mol}$

and for the trans isomer,

- $A_{E}=25.00 \times 10^{4} s^{-1}$

- $E^{\ddagger}=190.83 \mathrm{~kJ} / \mathrm{mol}$

The preexponential factors defined as the intersects of the Arrhenius straight line with the vertical axis, are naturally no longer relevant in the two models using reactant energies. The values reported in [6] were obtained using the linear Arrhenius equation dependent on temperature only through $R T$ and without taking into account the contribution of enthalpies. Since the experiments giving these results were conducted at temperatures around 800 $\mathrm{K}(1 / T=0.00125)[6$, the Arrhenius slopes obtained in these conditions must be compatible with the reactant enthalpies at this temperature, which are, using the Kirchoff approach described above, $E_{Z}(800)=60 \mathrm{~kJ} / \mathrm{mol}$ and $E_{E}(800)=54 \mathrm{~kJ} / \mathrm{mol}$. The values of $E^{\ddagger}$ found from the two reciprocal reactions, 186 and $191 \mathrm{~kJ} / \mathrm{mol}$, are not very distant, but the activation energies however, are no longer those currently reported. The previous values were $E_{a Z}=264.6 \mathrm{~kJ} / \mathrm{mol}$ and $E_{a E}=271.9 \mathrm{~kJ} / \mathrm{mol}$. They are now $E_{a Z}=125.2 \mathrm{~kJ} / \mathrm{mol}$ and $E_{a E}=136.3 \mathrm{~kJ} / \mathrm{mol}$ at $800 \mathrm{~K}$.

\subsubsection{Using positive energies}

The same treatment with positive energies gives very similar results

- $A_{Z}=2.82 \times 10^{4} s^{-1}$

- $E^{\ddagger}=207.34 \mathrm{~kJ} / \mathrm{mol}$

and for the trans isomer,

- $A_{E}=27.00 \times 10^{4} s^{-1}$

- $E^{\ddagger}=214.28 \mathrm{~kJ} / \mathrm{mol}$ 


\subsection{Model II}

Using the hypothesis of reactant-specific energy distribution and positive energies, given that reactant energies are much higher than $R T$, the values of $E^{\ddagger}$ will naturally be much higher than the previous ones. Moreover, since calculations from the reciprocal reactions use multiplications, the determination of $E^{\ddagger}$ is expected to be much more sensitive to imprecision than the previous one based on subtractions. When incorporating the positive energies calculated above in the probabilistic rate constant of Eq.(5) and adjusting the slope of the plot in Arrhenius coordinates at $T=800 \mathrm{~K}$. One finds for the cis isomer,

- $A_{Z}=2.43 \times 10^{6} s^{-1}$

- $E^{\ddagger}=1906.53 \mathrm{~kJ} / \mathrm{mol}$

and for the trans isomer,

- $A_{E}=6.25 \times 10^{6} s^{-1}$

- $E^{\ddagger}=1849.28 \mathrm{~kJ} / \mathrm{mol}$

The kinetic data thus give different parameter values depending on the model, but without being able to provide a discriminating criterion. The comparison of kinetics and equilibrium data will allow this discrimination.

\section{Comparison of the data ob- tained from Arrhenius coordi- nates with the results obtained at equilibrium}

The results of [7] obtained with catalysis are remarkably precise and compatible with previous, more partial measurements, as for instance [10]. Fig.4C and D show the comparison between values of equilibrium constants $K_{Z \rightarrow E}$ obtained in [7] and the ratios of kinetic constants $k_{Z \rightarrow E} / k_{E \rightarrow Z}$ based on the values measured in [6] and interpreted as either (i) the linear Arrhenius equations reported in [6], (ii) the Arrhenius equations modified to include reactant energies (Model I) and (ii) the probabilistic equation where reactant energies are envisioned as mean values (Model II). All approaches confirm the predominance of the trans isomer at low temperatures and the accumulation of the cis isomer at higher temperatures, but none gives exactly the equilibrium results. Obviously and even in case of uncertainties on the values of energies, the model II is disqualified since it predicts that cis-2-butene is almost absent below $300 \mathrm{~K}$, contrary to the experimental measurements [10, 7], in which this isoform is clearly present from $100 \mathrm{~K}$. This difference is specific of the model II because it is due to the participation of the activation thresholds in equilibrium. As a matter of fact, using the kinetic model II, the presence of trans- 2 butene in equilibrium can be shifted at lower temperatures by lowering $E^{\ddagger}$ in the equations (not shown).
A

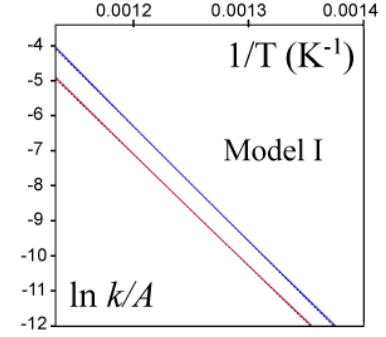

B

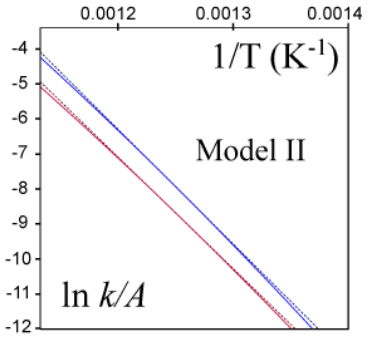

C

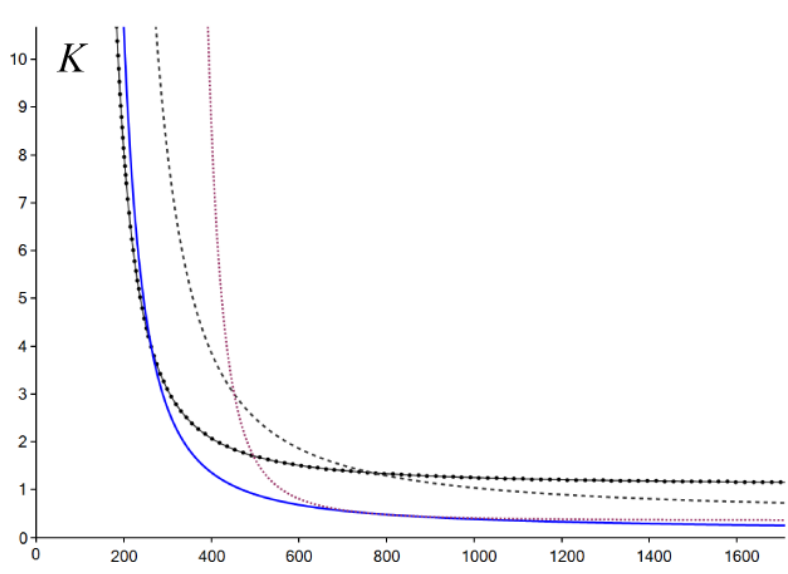

D

$\mathrm{T}(\mathrm{K})$

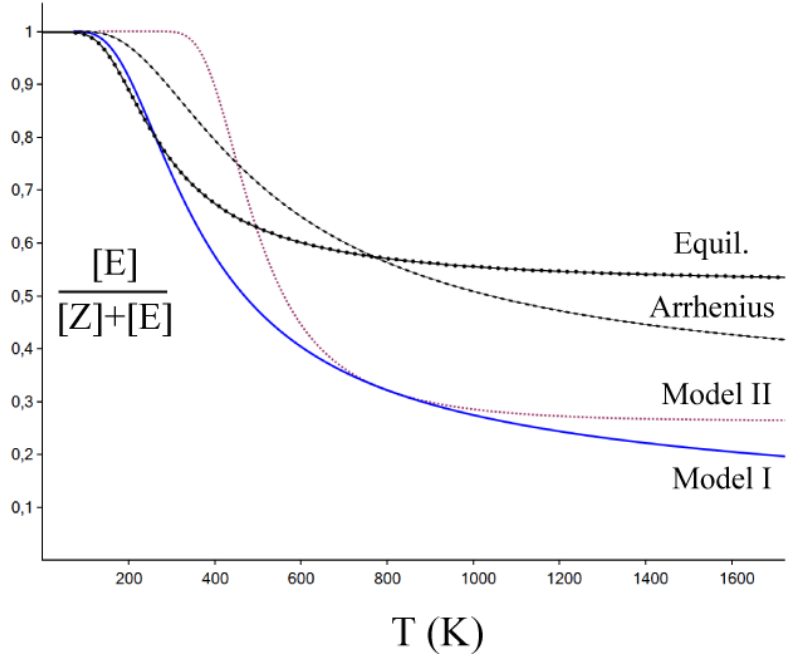

Figure 4. (A) Arrhenius plots of the reactions of 2-butene isomerization reported in [6] (dashed lines), superposed to those obtained by introducing reactant energies in the Arrhenius equation according to Eq.(8a). Cis to trans reaction in blue and trans to cis reaction in red. The theoretical parameters were obtained by adjusting the slopes at $800 \mathrm{~K}$. (B) Corresponding results when reactant energies are intro- 
duced through Eq.(5). (C) Comparison of the kinetic results obtained in Arrhenius coordinates [6] with the equilibrium constant [7]. (D) Proportion of the trans isomer in equilibrium predicted by the different theories. The curves shown in (C) and (D) are (i) the equilibrium data of [7] (black line with dots) in which the cis isoform is always predominant at all temperatures tested; (ii) the ratio of Arrhenius equations of rate constants $k_{Z \rightarrow E} / k_{E \rightarrow Z}$ of [6] (black dashed line, for which the switch between $Z$ and $E$ isomers occurs at 1000 $\mathrm{K}$ ); the curve predicted in the Model I (blue plain line, for which the equivalence between $Z$ and $E$ isomers is obtained at $468 \mathrm{~K}$ ); the curve predicted using the Model II (dotted line, equivalence between $Z$ and $E$ at $560 \mathrm{~K}$ ).

\section{Conclusions}

The comparison between kinetic and equilibrium experimental data shows that the hypothesis that the Arrhenius exponential factor could be a probability, does not apply to a chemical system of homogenized temperature. This model can apply to other physical situations like two-temperature systems or the evaporation of a clump of hot matter 2, but not to thermochemistry. The fundamental principle that activation energies do not influence equilibrium ratios is verified, but it is very instructive to return to the theoretical bases to see what this principle implies. This reductionist approach sheds light (i) on the nature of enthalpies which are not average energies but fixed basal values which can be increased by thermal fluctuations, and (ii) on the nature of the Arrhenius equation, which is a ratio of exponential probabilities calculated within a single energy distribution. This universal energy continuum encompasses all types of molecules, irrespective of our classification of the chemical world into different molecular species. Covalent bonds, which are used to define molecules, have no more importance for defining molecular energy than other features such as non-covalent bonds, stretching, rotations and vibrations. This view of molecular energy is in fact consistent with its immaterial nature, homogenizing between colliding molecules without any consideration for their structure. In this global exponential distribution, all forms are metastable and the most energetic molecules are the rarest ones. The acceleration of reactions by temperature is mediated at two levels: the increase of the denominator of the exponent $k_{B} T$, and the decrease of the numerator by reduction of the difference $E_{a}=E^{\ddagger}-H(T)$. Curiously this latter role of temperature is generally ignored in the interpretation of the Arrhenius equation as a straight line. The usual explanation of the role of temperature on reaction rates is based on a shift towards the high energies of a MaxwellBoltzmann distribution, which increases the fraction of molecules exceeding a fixed energy threshold. Strangely, this pedagogical interpretation completely holds for the hypothesis of reactant-specific mean energies disqualified here, but it is very partial under the hypothesis of the universal mean energy, since it does not take into account the enthalpy of the reactants. The introduction of reactant energy into the Arrhenius equation and the replacement of activation energies by energy thresholds, which are common to the two reciprocal reactions, would allow to anticipate the nonlinearity of Arrhenius plots. The simple knowledge of heat capacities allows to estimate this energy and to predict many kinetic and thermodynamic behaviors.

Acknowledgements. I thank very much Bill Kaiser and Freek Kapteijn for helpful discussions.

\section{References}

\section{References}

[1] Laidler K.J., The development of the Arrhenius equation, J. Chem. Educ. 61 (1984) 494-498.

[2] Michel D., Simply conceiving the Arrhenius law and absolute kinetic constants using the geometric distribution, Phys. A Stat. Mech. Appl. 392 (2013) 4258-4264.

[3] Michel D. A probabilistic rate theory connecting kinetics to thermodynamics. Phys A. 503 (2018) 26-44.

[4] Einstein A, Die Plancksche Theorie der Strahlung und die Theorie der spezifischen Wärme, Annalen der Physik 22 (1907) 180-190.

[5] Planck M, On the law of distribution of energy in the normal spectrum. Ann. Phys. 4 (1901) 553-563.

[6] Kaiser E.W., Pierce D.S. Study of the thermodynamics (Thermal and $\mathrm{Cl}$ Catalyzed) and kinetics of the cis and trans isomerizations of $\mathrm{CF} 3 \mathrm{CF}=\mathrm{CHF}, \mathrm{CF} 3 \mathrm{CH}=\mathrm{CHCF} 3$, and $\mathrm{CH} 3 \mathrm{CH}=\mathrm{CHCH} 3$ in $100-950$ torr of $\mathrm{N} 2$ diluent at 296$875 \mathrm{~K}$ : Effect of $\mathrm{F}$ and CF3 substitution on the isomerization process including the fluorine "cis effect". J. Phys. Chem. A. 119 (2015) 9000-9017.

[7] Kapteijn F., van der Steen A.J., Mol J.C. Thermodynamics of the geometrical isomerization of 2-butene and 2-pentene. J. Chem. Thermod. 15 (1983) 137-146.

[8] Selected values of properties of chemical compounds, Thermodynamics Research Center, Texas A\&M University, College Station, Texas, 1997.

[9] Prosen, E.J., Maron, F.W., Rossini, F.D., Heats of combustion, formation, and insomerization of ten C4 hydrocarbons, J. Res. NBS 46 (1951) 106-112.

[10] Scott R.B., Ferguson W.J., Brickwedde, F.G. Thermodynamic properties of cis-2-butene from 15 to $1500 \mathrm{~K}$. Journal of Research of the National Bureau of Standards, Volume 33 (1944) RP1592. 


\section{Appendices}

\section{A Assembly of single resonator heat capacities}

Real molecular heat capacities differ from the theory of Einstein in that they do not derive from a single wavelength of absorption. A first attempt of construction of the global heat capacity of a molecule may consist in juxtaposing its internal resonators. The so-called NeumannKopp law stipulates that the heat capacity of a composite material is equal to the weighted average of the individual elemental heat capacities. Using this postulate, the measured heat capacities can be adjusted to a theoretical curve of the form

$$
f(T)=A+\frac{B}{n} \sum_{\lambda_{1}}^{\lambda_{n}} \mathcal{C}\left(\lambda_{j}, T\right)
$$

where $\mathcal{C}\left(\lambda_{j}, T\right)$ is the normalized shape of the Einstein capacity for the chosen wavelength $\lambda_{j}$, ranging from 0 to 1 when either $\lambda$ and/or $T$ go from 0 to infinity.

$$
\mathcal{C}\left(\lambda_{j}, T\right)=\left(\frac{h c}{k_{B} T \lambda_{j}}\right)^{2} \frac{\mathrm{e}^{\frac{h c}{k_{B} T \lambda_{j}}}}{\left(\mathrm{e}^{\frac{h c}{k_{B} T \lambda_{j}}}-1\right)^{2}}
$$

and $A, B$ are constants aimed at adjusting the scale of $\mathcal{C}$ to the actual values of $C_{p}$, notably by taking into account the non-negligible values of heat capacities measured near $T=0$. Using the infrared absorption wavelengths reported for the two isoforms of 2-butene in the NIST Chemical WebBook, this averaging approach gives

$$
C_{E}=61+213[\mathcal{C}(3.5 \mu \mathrm{m})+\mathcal{C}(7 \mu \mathrm{m})+\mathcal{C}(10.5 \mu \mathrm{m})] / 3
$$

and

$C_{Z}=18+255[\mathcal{C}(3.5 \mu \mathrm{m})+\mathcal{C}(7 \mu \mathrm{m})+\mathcal{C}(10.5 \mu \mathrm{m})+\mathcal{C}(16 \mu \mathrm{m})] / 4$

This equation is satisfactory for trans-2-butene, but is less successful for cis-2-butene (Fig.A1C). Although this isoform presents two additional absorption wavelengths compared to the cis counterpart (around 15 and $17 \mu \mathrm{m}$ ), the best result was in fact obtained using only one wavelength, of intermediate value $16 \mu \mathrm{m}$. This method however yields long and hardly tractable equations. In addition, it is moderately justified because a molecule cannot be approximated as a juxtaposition of resonators since they are non-independent and likely to cooperate. The different peaks of the infrared absorbance spectrum may be involved in different molecular phenomena including $\mathrm{C}-\mathrm{H}, \mathrm{C}-\mathrm{C}$ and $\mathrm{C}=\mathrm{C}$ bond stretching or rocking, which are not independent from each others. Hence, it may be preferable to use a more arbitrary but simpler formula such as the simple exponential function used in the main text and the polynomial function shown below, useful for intermediate temperatures.

\section{B Polynomial fitting function for heat capacities between 273 and $1500 \mathrm{~K}$}

$$
C_{p} \approx \alpha+\beta T+\gamma T^{2}+\delta T^{3}+\epsilon T^{4}
$$

For the cis isomer, $\alpha=7.63, \beta=31.35 \times 10^{-2}$, $\gamma=-1.63 \times 10^{-4}, \delta=4.08 \times 10^{-8}$ and $\epsilon=-4 \times 10^{-12}$. For the trans isomer, $\alpha=-6.21, \beta=34.13 \times 10^{-2}$, $\gamma=-1.86 \times 10^{-4}, \delta=4.91 \times 10^{-8}$ and $\epsilon=-5.07 \times 10^{-12}$. 
A
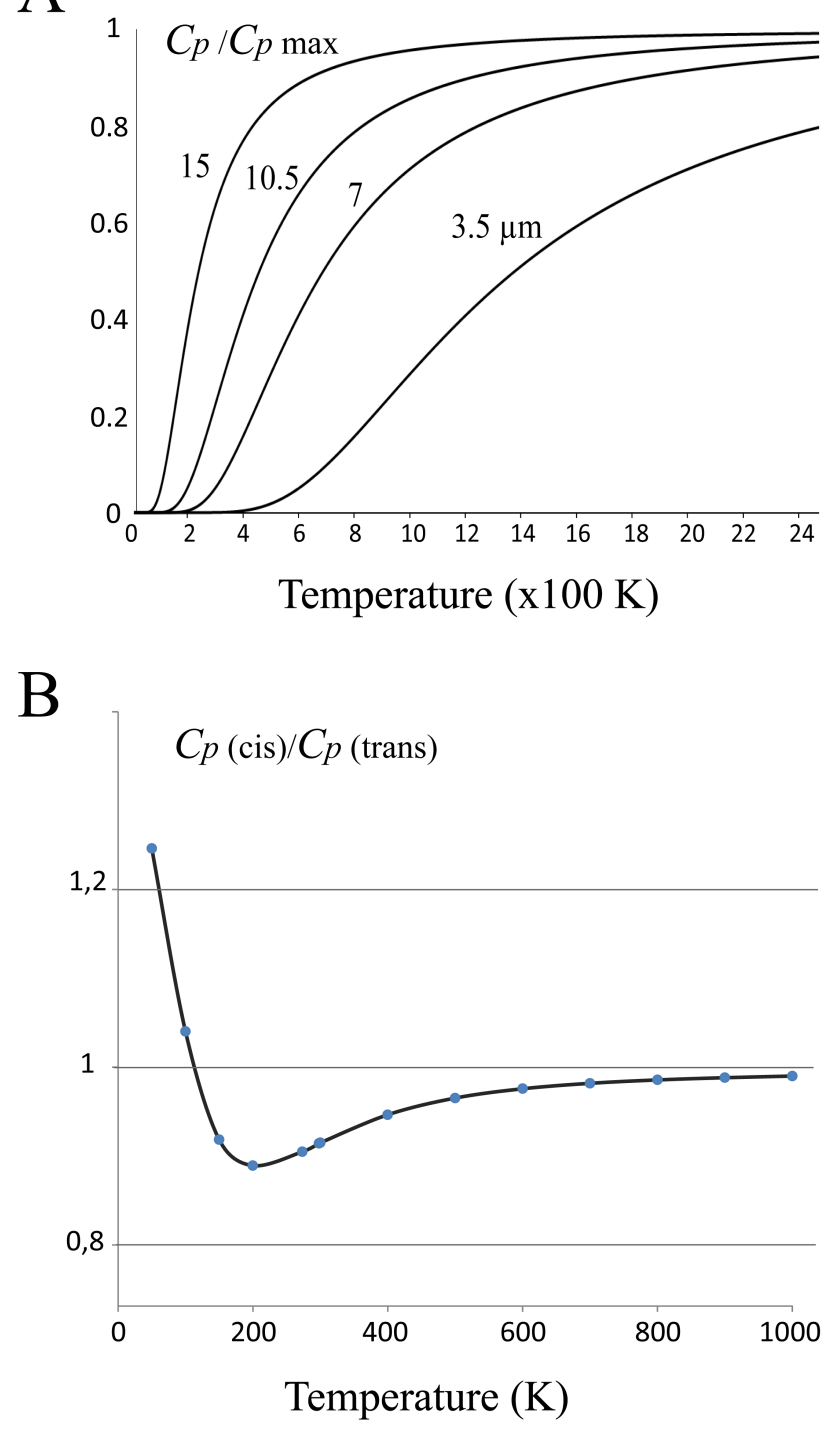

C

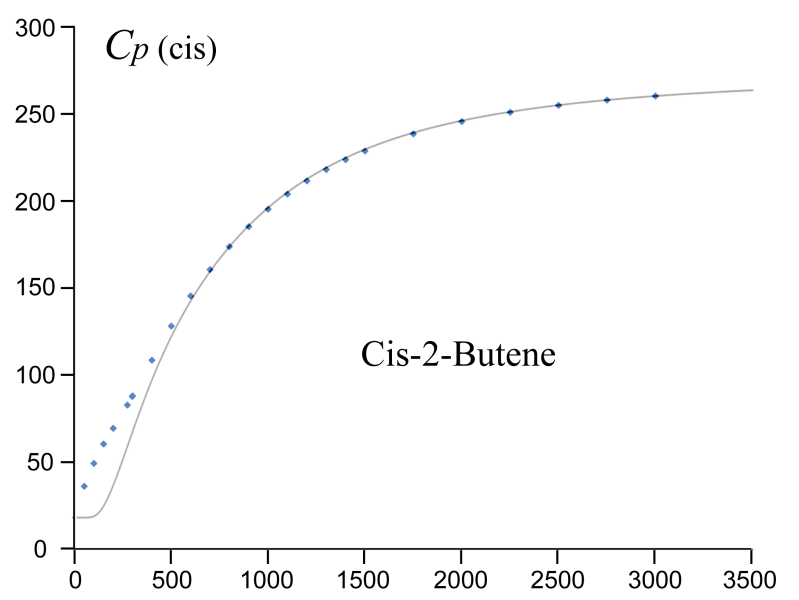

Temperature $(\mathrm{K})$

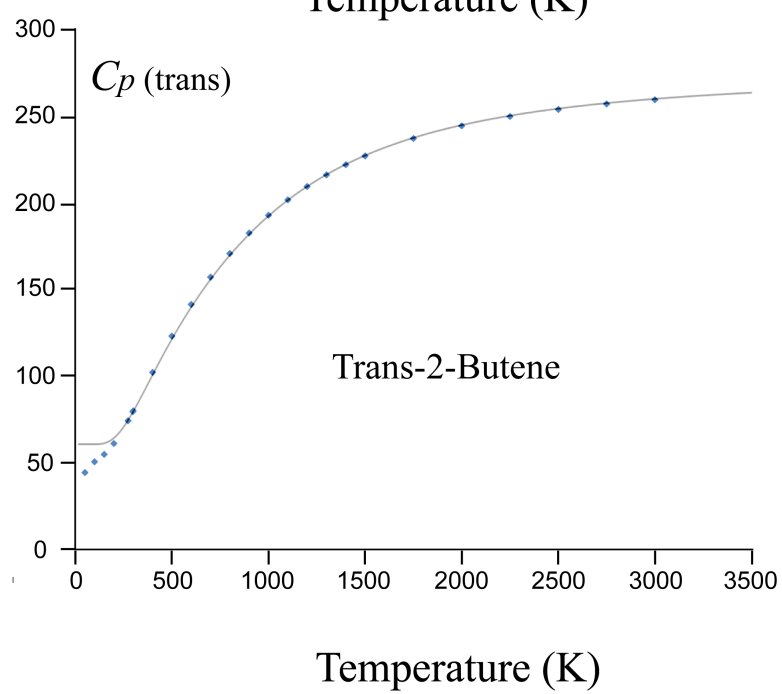

Figure A1. Heat capacities of the cis and trans isomers of 2-butene. (A) Normalized Einstein heat capacities (Eq.(A.2)) for the absorbed infrared wavelengths of cis-2-butene taken individually. (B) The complexity of real heat capacities is particularly evident at low temperatures, where the relative values obtained for the two isomers of 2-butene vary surprisingly. (C) Adjustment between the measured heat capacities, and functions defined as the sum of elementary heat capacities. Adjustment is good at high temperatures but not at low temperatures. 